\title{
Moral Injury in Times of COVID-19
}

\author{
Roger D. Williams • Jessica A. Brundage • Erin B. Williams
}

Published online: 2 May 2020

(C) National Register of Health Service Psychologists 2020

\begin{abstract}
Health care providers are facing increased risk of moral distress during the COVID-19 pandemic because of changes in clinical practice and resource allocation procedures. Health care systems that employ a proactive approach in mitigating the lasting effects of moral distress will fare better in the long run. Psychologists are well poised to apply timely psychological first aid to the crisis needs of patients, colleagues, and even themselves. Key interventions are detailed, including creating and promoting safety, calmness, connectedness, self-efficacy, and hope.
\end{abstract}

\section{Clinical Vignette}

Franklin, a 60-year-old, married, father of three children, was admitted as a person under investigation (PUI) for likely COVID-19 infection. He has multiple comorbidities including long-term tobacco use, congestive heart disease, chronic obstructive pulmonary disease, and diabetes. Upon admission, he was found to be declining rapidly with increased oxygen need and possible escalation of care to intubation with mechanical ventilator support. His spouse was not allowed to be at bedside because of the medical center's recently implemented infection control "no visitors" policy. The ICU physician told the patient's wife that there was a shortage of ventilators and that the triage team had made a negative escalation-of-care decision regarding her husband's treatment. She began begging to see her husband. As the team psychologist, you were asked to calm her and provide compassionate support. You had previously participated in the facility's scarce resource allocation discussions, and you know that her husband will die without assisted ventilation. The patient's wife began wailing from the first sentence of your conversation, which is agonizing to hear. She tells you that she has been quarantined at home alone for the past 12 days and she desperately wants to see her husband. You provide empathic listening as you grapple with the realities of the couple's separation during his final hours. Despite her incessant pleading, it is critical that you abide by the visitation policy resulting from the hospital's escalation of care model and standardized treatment protocol. This was the third case of the day for which you had been forced to "hold the line" on the nocontact policy. What is it costing you to do this work? Will it ever end? Will it have long-term effects on you?

\section{Clinical Challenge}

\section{Moral Distress}

Being forced to make clinical decisions, based on resource scarcity, that are inconsistent with therapeutic values is painful and distressing. Knowing that one patient will receive lifesustaining treatment while another will be denied that same treatment takes a significant emotional toll on the care provider. Being constrained by circumstances and system-issued protocols can cause an assault on the health care provider's moral core and foundational values. Jameton (1984) defined this as "moral distress"- the experience of knowing the right thing to do while being in a situation in which it is nearly impossible to do it.

Moral distress is likely to occur in acute health-care crises because the ability to provide optimal care is not absolute, but most often relative. Just because a ventilator or a necessary medication is available does not mean there is equal access for all, particularly during a crisis. The allocation choices made may result in dire consequences for some patients. These are real lives. Conversations must occur with patients about the treatment (or nontreatment) decisions, as well as with the families who will suffer the pain and loss. The health care provider's sense of responsibility is substantial whether or not the individual participated in the resource allocation decisions or had to uphold the decisions with the patient and family. Conflicts of interest, expressed and unexpressed biases, institutional constraints, limited resources, and personal values can become at odds.

Serving others is a fundamental value in the health care professional's pursuit of education, training, and career choice. Altruistic goals can become overshadowed by the realization of significant personal cost in time of crisis. During 
the COVID-19 pandemic, hospital psychologists are faced with the risk of illness or death of the patient as well as personal health risk. This also includes the realization of being an inadvertent carrier of disease to one's family. It is an emotional challenge to be forced to choose between your professional values and duties and the fundamental priority to protect our family from harm. This moral distress touches the deepest core of one's being. It can disrupt family systems and functional routines. Some health care workers have opted to live separately from their families, either alone or with coworkers, to limit potential virus exposure to loved ones. Others may not have that option.

Moral distress has been known to manifest as physical ailment and/or psychological suffering. Muscle tension, headaches, gastrointestinal upset, and fractured sleep are common. Feelings of exhaustion, frustration, helplessness, guilt, shame and worry are experienced by many health care providers. Sustained distress can evolve into moral injury, which is an erosion of trust in self, leadership, and the system as a whole (Shay, 2014). Perceived loss of ethical integrity can diminish one's personal and professional identity. Therapeutic effectiveness can become compromised and isolation from professional colleagues more likely. Moral injury for the health care provider can lead to burnout and even the decision to leave the profession altogether.

\section{Contextual Constraints}

Care provision is most commonly based on patient need and within the bounds of available insurance coverage. This was true on any routine day in 2019 in most U.S. hospitals, clinics, and private practices. If an insurance policy did not cover a particular procedure or medication, another option was found that did. Supplies were more abundant, and there was minimal rationing of care, services, and supplies.

The COVID-19 pandemic has fundamentally changed both practice and care decisions. National news stories highlight health care workers lacking sufficient personal protection equipment (PPE), such as being restricted to one gown and one mask per shift. Graphs and charts flood social media with numbers of needed ICU beds and the limited number of ventilators available. Washington State was headlined with these shortages in March, New York City in April, and one wonders which cities it will be in May. Will there be more beyond?

\section{The Ethical Dimension}

The COVID-19 pandemic response will continue to benefit from psychologists' involvement in scarce resource allocation decisions. These decisions occur on many levels whether within a medical institution or in a private practice setting. Institutional policies, prevailing norms regarding terminally ill patients, institutional limitations, staffing availability, equipment, and resource capability have major influence on decision-making.

Not surprisingly, a tug-of-war between individual health ethics and public health ethics arises during pandemics. Tensions are experienced between the needs of individual patients versus the community, patient-centered care versus the common good, and patient preference versus fair resource distribution. These disparate factors occur at all levels within the health care delivery system. Emergency departments, primary care practices, specialty services, and rehabilitation and long-term care programs must adjust service provisions to meet the changing treatment demands of the pandemic. Standard operating procedures are modified, and some longstanding operations must be discontinued entirely.

Times of crisis bring to light the challenging realities of maintaining ethical decision-making. Administrators, managers, and team leaders are forced to make decisions that are both informed and participatory. Yet, decision-making during a crisis typically does not permit lengthy gathering of all of the facts and all stakeholder views. A decision needs to be made, immediately.

Best practice would dictate employing a logical and valuebased decision-making process. These preemergency operational plans for use in crisis situations can prove invaluable. Options are weighted based on organizational values, shortand long-term consequences, benefit outweighing harm, applicability of established precedents to similar cases, and rationale for how decisions are made is apparent. Ethical leadership in crisis planning is a good guide for facilities delivering health care amid a pandemic. It is imperative that the psychological health and well-being of those required to implement crisis plans are addressed at all levels, from the decision-makers down to the patients served.

\section{Rational and Ethical Scarce Resource Allocation}

A rational model for allocating health care resources is imperative. Fortunately, many facilities have emergency plans with standardized review processes. These often-aspirational policies are met with the unforeseen realities of crisis, which demand real-time adjustments. Resource capacity extends beyond limited equipment, operating systems, and physical structures. The foundational goal of resource allocation is justice and fairness, the greatest good for the greatest number of people, consistently applied. This is best accomplished through transparent, reasonable, feasible, legal, and practical means.

Some scarce resource allocation systems have used unfair criteria such as age, disability status, pay source, and other biased and inappropriate factors. Psychologists provide helpful training for identifying biases, particularly social justice factors that result in some groups being 
disproportionally targeted for denial of treatment. An example is the growing evidence of racial/ethnic disparities in treatment decision. The City of Chicago (2020) reported the highest prevalence of infection and mortality in Black nonLatinx residents among all racial/ethnic groups. Compared to White non-Latinx residents, Black non-Latinx residents had double the prevalence of infection $(23.6 \%$ vs. $52.2 \%$, respectively) and mortality ( $1.8 \%$ vs. $4.1 \%$, respectively).

There are clear benefits from scarce resource processes, if resources are distributed fairly and services are provided equitably. When crisis standards of care are implemented systematically, allocation plans ensure ethical quality of care, promote accountability, reduce liability risk, potentially lower the risk of moral distress, and provide a feasible road map from the chaos inherent in the crisis. Allocation plans can include mechanisms to evaluate outcomes and adjust distribution based on resource availability. One well-recognized resource is "Meeting the Challenge of Pandemic Influenza: Ethical Guidance for Leaders and Health Care Professionals in the Veterans Health Administration" (Pandemic Influenza Ethics Initiative Work Group of the Veterans Health Administration's National Center for Ethics in Health Care, 2010).

Scarce resource allocation processes that only assess administrators' risk obscure the patients' psychological needs and well-being. Similarly, processes that solely account for frontline caregivers may be fraught with personal reactions and prejudices. Several health care systems have adopted the VHA Ethics Center guidance (noted above) regarding standard of crisis care. It guides implementation of triage protocol by emphasizing survivability rather than use of arbitrary exclusion criteria such as age, disability status, or ability to pay.

\section{Psychological First Aid}

The "human factor" has traditionally been missing in scare resource allocation business models. It is critical that choices are made while balancing the needs of the patient with system constraints and adopted protocols. COVID-19 underscores the shared risk for systems, but also for patients and health care providers alike. The psychological cost of making such life and death decisions can be profound for the individuals involved.

Health care systems are rapidly developing and implementing support systems to address the unique demands placed on providers during the COVID-19 pandemic. A proactive approach grounded in "psychological first aid" principles can partially mitigate the effects of moral distress in the short- and long-term (Brymer et al., 2006; Epstein \& Hamric, 2009). Psychologists can apply psychological first aid principles to bolster their own coping efforts. They can do the same in their interactions with patients and colleagues, which will foster moral resilience across the health care continuum.
Psychologists are uniquely positioned to develop and provide education and interventions within the larger organization or the community.

\section{Create a Sense of Safety}

Attending to safety concerns in times of crisis is fundamental. This involves using strategies that target both emotional needs and physical safety. Psychologists can disseminate resources to assist patients and colleagues, as well as model healthy sleep, proper nutrition, regular exercise, and stress management techniques. They can engage individuals who are struggling in brief problem-solving exercises. Individuals will then be able to identify and eliminate modifiable barriers that are interfering with goal attainment. Mutual problem solving can be effective in a casual conversation with coworkers in the hallway or in formal psychotherapy with a patient.

Methods for conveying safety in clinical contexts have transformed in light of the COVID-19 pandemic. Working behind the barriers of face shields/masks and other required contact isolation gear increases the sense of distance between provider and patients as well as between colleagues. It feels unnatural to deliver health care services within these confines. Face shields/masks are hot and uncomfortable, especially with extended use. Simply acknowledging the frustration associated with these constraints can create a shared experience and a safer emotional landscape. In the outpatient setting, access to telepsychology services during the COVID-19 crisis has grown exponentially, with $80 \%$ of practicing psychologists having moved their practices online (Sammons et al., 2020). The shift from in-person care to email, telephone, and video contact has necessitated changes in how psychologists establish rapport. The intersection of technology and psychotherapy can be challenging because of equipment availability and access to technology. When technology is available, attention must be given to ensuring adequate connection bandwidth, monitor resolution, camera and microphone placement, sitting position, and the focal point on the screen for natural eye contact. All of these aspects of telehealth provision can foster or compromise a sense of safety and support (Glueck, 2013).

\section{Cultivate Calm}

Practicing mindfulness and teaching patients and colleagues these techniques promotes emotion regulation and reduces depression and anxiety (Hempel et al., 2014). Two simple tools require only 30-60 s: Mindful breathing and centering presence can be used during a shift or to help ease the transition from work to home. Mindful breathing involves slowly inhaling through the nose, and exhaling through the mouth, while focusing one's attention on the bodily sensation of the 
breathing process (and letting all other thoughts go). Building a centered presence involves pausing briefly before entering the next patient encounter, taking a few long, slow, deep breaths, letting the thoughts of the last encounter drift away, and calmly orienting self to the present (Norcross \& VandenBos, 2018).

Identifying physical quiet spaces for staff to decompress and recharge during shift breaks normalizes that downtime is necessary amid stressful working conditions. Having mental health staff available to help frontline providers process the demanding and unfamiliar situations in crisis care can help them regain their composure and focus. Psychologists can also assist colleagues by providing other healthy ways to relieve pent-up energy, which contributes to agitation and detracts from efficiency and effectiveness at work and at home.

\section{Foster Connectedness}

Psychologists have a proclivity of fostering connectedness. It is important to recognize that social distancing is not commensurate with professional isolation. We are all in this together. We cannot underestimate the power of being present, showing support, and empathizing through ever-evolving practice during a crisis. Increasing caseloads require complex treatment approaches, grieving relatives are deprived of contact, and personal suffering within one's family can result in pervasive desensitization. It is important to build routines that emphasize mutual understanding of each other's struggles and contributions. It is particularly critical for leaders at all levels to voice their appreciation of staff as often as possible. This outward praise can strengthen the connection between providers and patients. Likewise, being able to express concerns or distress can have utility, allowing individuals to tap into the shared experiences of personal suffering, and one's imperfections can become critical points for self-kindness that strengthen empathy when helping others (Nouwen, 2010).

Virtual peer-led support groups are another way for staff to connect, commiserate, and bolster resilience through normalization and shared problem solving. Psychologists are ideal facilitators for these support forums. Dialog about the clash between clinical ethics and public health ethics may be common themes in these groups. Knowing these ethical principles and how they are impacting scarce resource allocation and triage protocols in hospital settings can bolster efforts to resolve moral distress when violations occur. For instance, when a patient's treatment preference to use mechanical ventilation cannot be upheld because of standardized triage decisions, identifying the principles in conflict (autonomy vs. justice) becomes an important first step in understanding why feelings of guilt might surface. Subsequently, reconstructing more flexible moral schemas that integrate contextual factors can reduce a sense of responsibility and promote healing (Litz et al., 2009).

\section{Support Self-Efficacy}

The onslaught of health care stressors during a pandemic, like those experienced in military combat, results in substantial personal and professional loss, pain, and injury. When under duress, leaders in systems may slip into harsh reactions to negative patient outcomes. Organizational responses can cause providers and psychologists to feel helpless in the face of growing tragedy. Psychologists are susceptible to this, too. Guilt and blame associated with moral distress can undermine self-confidence. Identifying and evaluating these feelings through mindfulness activities and consultation with colleagues can lay the groundwork to challenge cognitive (mis)attributions that have contributed to these emotions.

Redirecting attention and focusing energy on what one can control improves quality of patient care and reduces negative outcomes. Although the format may vary, delivering evidence-based interventions to patients can be a powerful reminder of eventual benefit while in the midst of overwhelming circumstances. Psychologists' collegial interventions can lessen the burden of staff shortages and difficult work conditions. Psychologists can benefit by anchoring themselves in the intrinsic value gained from clinical activities, especially those that provide a sense of joy and confidence, bolstering satisfaction.

\section{Nurture Hope}

Reconnecting with personal values and a mission can provide a sense of purpose in the midst of the health care crisis. A mission can be adopted from the organization in which the psychologist works, or an expressed aim of the patients being treated, or even a personal creed of the individual provider. Printing a copy of the mission and displaying it where it can regularly be seen and read is a helpful reminder, especially in times of despair. Imagining the future in a positive way and sharing this vision with patients, colleagues, and leadership can birth hope.

Additionally, instilling hope in work rituals can have a positive effect. Rather than having staff simply leave when their shift is completed, perhaps invite them to meet briefly in a common area that allows reasonable social distancing, maybe with coffee or tea, to share a positive or heartwarming event that occurred during the shift. Each person can be asked to share something for which they are grateful, whether it is something at work or in their personal lives. This is an example of translating positive psychology interventions into collective debriefing and mutual support activities. 


\section{Wrapping up: Practical Tips for Supporting Psychological Health}

The COVID-19 pandemic has generated many sources of stress and subsequent distress for health care workers, patients, and families. It is critical to be aware of the various feelings that may arise and try not to hide emotional reactions. Psychological first aid tenets provide a road map that fosters resilience for patients, providers, and health care systems to navigate the ethical dimensions of the COVID-19 pandemic.

- Acknowledge stress, pressure, and sacrifice. Acknowledgement from leaders and peers is vital for normalizing staff reactions and knowing that our experiences are shared.

- Adopt an ethical mindset. Early awareness of two or more ethical principles being at odds can cause moral distress and if recurrent, moral injury. Be proactive to mitigate harmful consequences.

- Lean on colleagues. Find ways to dialog through video chat, telephone, and email. Talk about your reactions and the distress you are experiencing. If moral distress - $\mathrm{a}$ seemingly impossible conflict between values and actions - arises, talk about it.

- Connect with patients. Create empathic interactions despite current barriers to usual care (e.g., social distancing, PPE, intervention type). Delivering evidence-based interventions promotes self-efficacy and reminds health care providers to not give up in the midst of overwhelming circumstances.

- Bolster resilience. There is continual need for resources that foster moral repair and resilience. Without such resources, personal guilt will erode professional confidence. Realize that you, your colleagues, and other health care providers may grapple with the moral residue of COVID19 beyond its actual period of immediate threat.

\section{References}

Brymer, M., Jacob, A., Layne, C., Pynoos, R., Ruzek, J., Steinberg, A., Vernberg, E., \& Watson, P. (2006). Psychological first aid: Field operations guid (2nd ed.). National Child Traumatic Stress Network and National Center for PTSD. Retrieved from https:// www.nctsn.org/resources/psychological-first-aid-pfa-fieldoperations-guide-2nd-edition

City of Chicago. (2020, April 6). Latest data. Retrieved from https:// www.chicago.gov/city/en/sites/covid-19/home/latest-data/2020-0406.html
Epstein, E., \& Hamric, A. (2009). Moral distress, moral residue, and the crescendo effect. Journal of Clinical Ethics, 20, 330-342.

Glueck, D. (2013). Establishing therapeutic rapport in telemental health. In K. Myers \& C. L. Turvey (Eds.), Telemental health: Clinical, technical, and administrative foundations for evidence-based practice (pp. 29-46). Elsevier.

Hempel, S., Taylor, S. L, Marshall, N. J., Miake-Lye, I. M., Beroes, J. M., Shanman, R., Solloway, M. R., \& Shekelle, P. G. (2014). Evidence map of mindfulness. VA-ESP Project \#05-226. Health Services Research \& Development Services, Department of Veterans Affairs.

Jameton, A. (1984). Nursing practice: The ethical issues. Englewood Cliffs, NJ: Prentice-Hall.

Litz, B. T., Stein, N., Delaney, E., Lebowitz, L., Nash, W. P., \& Silva, C. (2009). Moral injury and moral repair in war veterans: A preliminary model and intervention strategy. Clinical Psychology Review, 29, 695-706.

Norcross, J. C., \& VandenBos, G. R. (2018). Leaving it at the office: A guide to psychotherapist self-care (2nd ed.). New York: Guilford Press.

Nouwen, H. J. M. (2010). The wounded healer (2nd ed.). New York: Image Doubleday.

Pandemic Influenza Ethics Initiative Work Group of the Veterans Health Administration's National Center for Ethics in Health Care. (2010, July). Meeting the challenge of pandemic influenza: Ethical guidance for leaders and health care professionals in the Veterans Health Administration. Retrieved from https://www.ethics.va.gov/ docs/policy/meeting the challenge of pan flu ethical guidance vha_2010_web_posting_2013.pdf

Sammons, M. T., VandenBos, G. R., \& Martin, J. (2020). Psychological Practice and the COVID-19 Crisis: A Rapid Response Survey. Journal of Health Service Psychology, 46, yyy-zzz.

Shay, J. (2014). Moral injury. Psychoanalytic Psychology, 31, 182-191. https://doi.org/10.1037/a0036090

Publisher's Note Springer Nature remains neutral with regard to jurisdictional claims in published maps and institutional affiliations.

Roger D. Williams, $\mathrm{PhD}$, is a licensed psychologist in the Spinal Cord Injury \& Disorders Center, Zablocki VA Medical Center (ZVAMC). He is the ZVAMC ethics consult co-coordinator as well as a member of the Scarce Resource Allocation Team and Integrated Ethics Council. He is also an associate professor in the Department of Psychiatry and Behavioral Medicine, Medical College of Wisconsin.

Jessica A. Brundage, $\mathrm{PhD}$, is a licensed psychologist in the Spinal Cord Injury \& Disorders Center, Zablocki VA Medical Center (ZVAMC). She serves on the Ethics Consultation Service at ZVAMC. She is also an assistant professor in the Department of Psychiatry and Behavioral Medicine, Medical College of Wisconsin.

Erin B. Williams, $\mathrm{PhD}$, is a licensed psychologist in the Primary Care Mental Health Integration program, Zablocki VA Medical Center. She is a crisis intervention trainer for first responders, police and sheriff departments, correction officers, crisis negotiation teams, and federal agents in Wisconsin. 